\title{
Adapting the Battery Model in the Mission Planning System of Ageing Satellites
}

\author{
F. Stathopoulos ${ }^{1}$ \\ LSE Space GMBH, Argelsrieder Feld 22, 82234 Weßling, Germany \\ C. Lenzen ${ }^{2}$ and F. Mrowka ${ }^{3}$ \\ German Aerospace Center (DLR), Münchner Straße 20, 82234 Weßling, Germany
}

\begin{abstract}
In June 2017 the 10th and the 7th anniversaries of the TerraSAR-X and TanDEM-X satellites respectively were celebrated. Both have exceeded their design-lifetime in orbit being both in great condition and fully operational. The inevitable ageing of the satellites is the main driver for the optimization of the batteries-utilization in the joint TerraSAR-X / TanDEM-X Mission Planning System. In addition, the sun-synchronous "Dawn-Dusk" orbit of the satellites and the different flying formations, together with dedicated acquisition campaigns, enforce the TerraSAR-X/TanDEM-X Mission Planning System to be adapted in order to face the new challenges that are rising. In this paper we describe all actions taken in the TerraSAR-X/TanDEM-X Mission Planning System along with the strategies foreseen for the future, in order to cope with the energy model of the ageing batteries of the TerraSAR-X and the TanDEM-X satellites, continuing at the same time the automated and unattended Mission Planning operations.
\end{abstract}

\section{Nomenclature}

$\begin{array}{lll}\text { DLR } & = & \text { German Aerospace Center } \\ M P S & = & \text { Mission Planning System } \\ P T S & = & \text { Power and Thermal System } \\ \text { SAR } & = & \text { Digital Elevation Model } \\ \text { DEM } & = & \text { TerraSAR-X mission } \\ \text { TerraSAR-X } & \text { TerraSAR-X add-on for Digital Elevation Measurements mission } \\ \text { TanDEM-X } & = & \text { TerraSAR-X satellite } \\ \text { TSX } & = & \text { TanDEM-X satellite }\end{array}$

\section{Introduction}

The TerraSAR-X and TanDEM-X mission consist of the TSX and TDX satellites, which are flying in bistatic close formation that build a single-pass space-born radar interferometer [1]. The SAR acquisitions require high peak power to transmit the radar beam towards the target area. The energy budget for the SAR acquisitions has an upper threshold per orbit, therefore it is required an optimum planning for the SAR acquisitions that are selected to be executed, respecting all operational aspects. In the joint TerraSAR-X / TanDEM-X Mission Planning System [2] exists a well-defined energy model [3] which is considered during scheduling the activities of the satellites.

In parallel, the batteries of the TerraSAR-X and TanDEM-X satellites after more than ten and seven years in orbit respectively, are the modules which are affected the most by the ageing of the satellites. The battery performance depends on the load that is requested every time by the satellite bus or payload.

\footnotetext{
${ }^{1}$ Subsystem Operations Engineer, Mission Operations Department, German Space Operations Center (GSOC).

${ }^{2}$ Mission Planning System Engineer, Mission Operations Department, German Space Operations Center (GSOC).

${ }^{3}$ Mission Planning Manager, Mission Operations Department, German Space Operations Center (GSOC).
} 
In the last years, the Mission Planning team together with the Power and Thermal team, we study the behavior of the batteries over the performed activities, and we try to conclude to a formula that optimizes the battery utilization, respecting all safety limits and measures, while all requested operational activities are executed. Here we have to note that the batteries are in the expected state, and the values they reach are far from the thresholds that would trigger an onboard event. The objective of the studies is to get an insight into the power system of the satellites well in advance, optimize its usage and get prepared for the future while there is no particular stress by the system behavior.

In this paper we are presenting the actions taken in the last years in order to cope with the inevitable ageing of the batteries of the satellites, as well as with the strategy that is planned to be followed in the upcoming years.

\section{Power Constraints in the Mission Planning System}

The TerraSAR-X and TandDEM-X missions have been designed such that the TSX and TDX satellites have to follow the envisaged TerraSAR-X reference orbit characterized by a sun-synchronous dusk-dawn orbit with a repeat cycle of 11 days [1]. The orbit of the satellites is selected in a way that the satellites remain under the sun light throughout the mission. As a result, whenever high energy is requested for tasks to be executed on board, the energy is firstly supplied directly by the solar panels, and if needed provided by the battery.

Moreover there are some exceptional cases, when the solar panels are not pointing to the sun, and no energy can be supplied by the panels to the satellite. Obviously all the energy requested by the bus and the payload is provided only by the battery, and those cases attract our interest to study them, since the unregulated power request is much higher than during any other activity under nominal conditions. The two exceptions that the solar panels of the satellites are not pointing to the sun are presented below in addition to an indirect consequence to the stress on the batteries. The first is the yearly eclipses period when the satellites are in eclipse for a few minutes (up to twenty minutes) every orbit. The second is happening during acquisitions with a specific satellite attitude that takes the solar panels out of the field of view of the sun, the so called Left Looking Acquisitions. Finally the Exclusion Zones concept [1], which is turning the balance of the activities towards one of the satellites, might increase the energy request for a period of time in one of the two satellites. Those cases are presented in details below.

The higher the unregulated power request, the higher the energy and the current request it is, which leads to lower unregulated voltages. The lower limit for the battery safety, as defined by the manufacturer, is set on a voltage range that is determined under the discharge current in full load, considering the internal resistance of the batteries. We aim to prevent any high energy request that would drop the unregulated voltage in a state that would interrupt the support of the nominal satellite operations. As abovementioned we have never experienced such an event, with the lowest values currently being far from those limits. Nevertheless we are monitoring the unavoidable battery performance drop and target in considering it with the most accurate way in the Mission Planning System.

\section{A. Eclipses}

The sun-synchronous "Dawn-Dusk" orbit of the satellites includes a period of eclipses between April and August every year for a few minutes of every orbit. During an eclipse, the satellite is in the umbra of the earth and the solar panels cannot provide any energy to the satellite. The full energy required by the satellite bus and the payload is supplied exclusively by the batteries. As a result the stress on the batteries of each satellite during this period is considerably higher, particularly when several successive acquisitions are found within the same eclipse period, prior to the next battery recharge.

\section{B. Left-Looking Acquisitions}

A similar impact on the energy-request from the batteries, as during the eclipses, is caused by specific SAR acquisitions, which presume an attitude change that brings the sun out of the field of view of the solar panels, the so-called Left-Looking acquisitions. The Left-Looking acquisitions are executed at any time throughout the mission lifetime, but a peak can be noticed during dedicated campaigns. Here we have to note that depending on the period of the year, it can be that a Left-Looking acquisition brings the solar panels in partial shadow. Nevertheless during the summer months (when also the eclipses occur) a Left-Looking acquisition brings the solar panels in full shadow, and no energy is supplied to the spacecraft by the solar panels.

\section{Exclusion Zones}

An additional constraint that affects the batteries indirectly is the exclusion zones, a feature introduced after the launch of the TDX satellite, and the close formation flying together with the TSX satellite. In parts of the orbital 
evolution transmitting radar pulses from the one satellite would lead to illumination of the partner satellite, causing permanent damage to the SAR payload of the latter. As a consequence operational measures have been established in order to avoid radar transmission in these forbidden areas for safety reasons, called Exclusion Zones [1]. All SAR acquisitions in an Exclusion Zone can be executed only by the partner satellite of the one being in an exclusion zone. When an eclipse is overlapping with an exclusion zone the battery of the exclusion-zone-free satellite takes the entire load and obviously it is stressed more during this period. Depending on the flying formation of the satellites, it can be either the TSX or the TDX satellites that are in the exclusion zone while in eclipse.

A combination of the latter composes the worst case scenario for the battery model of the Mission Planning System: a series of consecutive Left-Looking acquisitions close or overlapping with an eclipse might block for a long timeframe the charging of the battery of the satellite that is out of the exclusion zone and in the only satellite of the two that can execute the SAR acquisitions. Such cases have been identified and analyzed by the Mission Planning team, in order to create a data set of examples and study the performance of the batteries together with the Power and Thermal team.

\section{Analysis and first Adaptations in the Mission Planning Operations}

Already in the previous years, the TerraSAR-X / TanDEM-X Mission Planning System team has performed thorough analyses on the workload and its distribution between the two satellites [4]. The goal of those analyses is to check, to assess and finally to feed back to the Mission Planning System the necessary information for the even distribution of the workload between the two satellites, based on the specific flying formation and acquisition campaign every time. Now an advanced concept is applied on the operational data, considering on top the stress of the batteries of each satellite into account. Statistics are performed on a 11 days cycle basis, assessing the distribution of the workload. The first measure taken

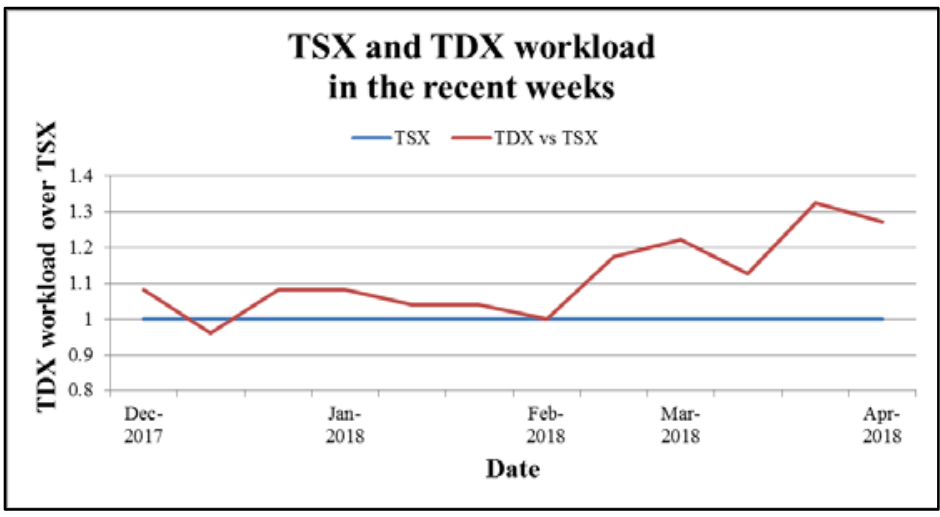

Figure 1. The workload of TDX satellite vs the one of TSX. Measures are taken in order to keep the workload balanced between the two satellites.

in order to drive the workload to the wished satellite was a (configurable) weighted randomization feature in the algorithm of Mission Planning. It has been introduced for selecting the satellite on which an acquisition is executed during every planning run. Via this feature, and based on the given input from the corresponding analyses, the Mission Planning System is able to manage and balance the workload distribution on the two satellites. The results are monitored continuously in order to make sure that the workload is balanced between the two satellites. Besides in case of changes in the satellite flying formation, where changes in the workload are anticipated, preliminary analyses are performed in advance in order to forecast the change in the workload, and be prepared for any operational actions to be taken.

\section{Power and Thermal input to the Mission Planning System}

The analyses of the Power and Thermal System team together with the contribution by the manufacturer team stated a secondary degradation factor on the Li-Ion batteries of the satellites [5,6], on top to the primary factor, which is the normal and expected ageing of the batteries. The primary factor has been studied since the beginning of the mission, and it has been considered in the Mission Planning System since its first implementation. The secondary factor is an indirect consequence of the ageing, and is identified as the Diffusion Rate Limitation on the batteries [5]. The Diffusion Rate is the current flow rate from and to the battery. The consequence of the Diffusion Rate Limitation is a temporary change in the current request by the battery, which impacts on the battery voltage. During high energy request, there is high current request in the battery, which leads to a reduction of the available 
voltage. Similarly an over-voltage might occur at the end of charge. The above analyses made it necessary for the Mission Planning System to adapt the power constraints it considers. The main objective to incorporate the new constraints in the Mission Planning System was the translation of the current request by the battery in terms of energy, in order to be embodied in the existing energy model of the Mission Planning System. A connection between the current request and the energy consumption had to be defined. The adaptations in the Mission Planning System are presented in the next paragraph.

\section{Adapting the Battery Model of the Mission Planning System}

All the above mentioned constraints, together with the inputs from the TerraSAR-X/TanDEM-X Power and Thermal System team for the "diffusion rate effect" in the Li-Ion batteries [1], have been incorporated in the TerraSAR-X/TanDEM-X Mission Planning System as new features that are considered during the joint planning of the timelines for the two satellites and the two missions [2]. Along the years both teams, the Power and Thermal and the Mission Planning Operations team investigate this topic and upgrade the approach on handling the activities from the power point of view. It can be obvious, that the focus is on the high energy demanding tasks, especially the SAR acquisitions and particularly during the cases described in paragraph III. Below we present the models that have been already developed in the Mission Planning System in order to cope with the challenges of the ageing batteries.

\section{A. Model 1}

The first model implemented in the Mission Planning System is based on the unregulated bus Voltage slope analysis performed by the Power and Thermal team [5,7]. The outcome of this analysis revealed the relationship between the discharge power and the voltage rate with a formula. This was the first step where the two worlds of the Power and Thermal System and of the Mission Planning System could be connected. As described in the relevant paper [5], the formula was introduced and was applied as a constraint check to every SAR acquisition considered by the Mission Planning System. Nevertheless, this formula could not consider more complex scenarios, such as consecutive SAR acquisitions in parallel to eclipses, while the partner satellite is in an exclusion zone. Therefore it was necessary to upgrade the handling of the high energy request activities, not only as individual tasks to be executed but as a group of activities. This lead to introducing the second model, which is described below.

\section{B. Model 2}

The second model is focusing on creating groups of activities planned by the Mission Planning System, in order to cope with the limitations of the first model implemented as described above. The implementation of this second model has been based on the already existing Energy Model of the Mission Planning System. In this Energy Model it is defined the power consumption of every regular activity, or it is requested as input for every activity with dynamic power consumption (i.e. the SAR acquisitions), and based on the planned activities the power consumption is projected over the time, creating an energy consumption timeline. This energy profile exists since the beginning of the mission, and has been proved quite accurate over the years of the mission while it is cross-checked against the energy profile derived by the satellites telemetry. Here we have to note that in this model the ageing of the satellites batteries is considered, with a degrading factor over the years. Nevertheless, the newly introduced Diffusion Rate Limitation could not be captured by the existing Energy Model, basically due to its temporary impact on the battery performance.

This topic was under investigation by the Mission Planning Operations team, in order to find out the factors that affect the performance of the batteries, focusing in the eclipses periods. We found out due to the fact that during an eclipse the battery is not recharged, the dominating factor of the battery voltage drop inside an eclipse is the total duration (sum of individual durations) of the SAR acquisitions inside this eclipse, as it can be depicted in Figure 2. Therefore we introduced the "chain" concept of SAR acquisitions inside an eclipse, in the so called "chain model". A (configurable) threshold was set to the maximum SAR acquisition duration inside an eclipse at 180 seconds [5]. A separate study was performed in order to verify the duration of the diffusion rate impact after a SAR acquisition. It was proved that after a few minutes without any SAR activity, the battery would perform nominally again, even if the satellite was still in Eclipse. Therefore in the model implementation we declared two different (and configurable) parameters. The first is the period that we would consider a sequential SAR acquisition as a chain to the previous acquisition. This parameter can be declared either as the whole eclipse period or with a time period after every SAR acquisition. The second, as abovementioned, is the threshold of maximum SAR acquisition chain activity allowed 
during scheduling the timeline. Every year, prior to the start of the eclipses period, a new study is performed by the Mission Planning Operations team together with the Power and Thermal team in order to define the values of the model's parameters.

Finally considering the worst case scenario, as defined above, which consists of acquisitions during eclipses and/or of series of consecutive Left-Looking acquisitions, it has been decided to process the Left-Looking SAR acquisitions the same as the eclipses, from the energy point of view. As a consequence they were also considered in the chains, applying exactly the same thresholds and limits as for the eclipses. Via this model, the Mission Planning System accounts the "diffusion rate effect" by controlling the maximum

allowable discharge energy for every payload operation supported by the batteries. The SAR acquisitions are now treated not only individually per acquisition but also as one special "super" event accumulating the energy consumption of all the acquisitions as long as the solar panels are not illuminated, either due to an eclipse or a Left-Looking spacecraft attitude.

Prior to their operational activation, all the newly introduced features were extensively tested and validated in order to fulfill a twofold target: a) respect the available energy budget, while b) narrow the impact on the number of the not-planned acquisitions due to the limitations that the new features imply. Under this optimization rationale a continuous review of the operational data is leading to the preparation of an updated energy model in the upcoming years, when the stress on the batteries is expected to be even higher.

\section{Further development}

Currently the model 2 (chain model) is operational on both satellites, TerraSAR-X and TanDEM-X. It is foreseen to be applied during the upcoming eclipses period of 2018. Continuous monitoring and analysis will be performed throughout this eclipses period. At the same time both the Mission Planning and the Power and Thermal teams are working on the further development of the existing models in order to deal with the battery degradation performance in the next years.

The chain model can be considered a statistical model of one variable, which is the duration of SAR activity inside a chain, or in other words: the time. In this model, time is expressed in two different terms, one in the threshold of the maximum payload activity, and the second in the period that a chain is still considered.

The further implementation, in a third model, is taking into account more parameters which could affect the battery performance during a SAR acquisition. Those parameters can be categorized in two groups: a) the SAR acquisition properties, i.e. the duration, the energy consumption, the power consumption; and b) the events prior to this SAR acquisition which can be concentrated in the available Energy and the Power Consumption at the start of a SAR acquisition. This is already depicted in the Energy Model of the Mission Planning System, as it was described above. In other words, we are developing a multivariable statistical model for the behavior of the battery.

This new model is still under development, but a first draft version will be tested offline (not operationally) during the upcoming eclipses period of 2018. The list of independent variables of the model is still not final; it could be updated while processing the test results.

The inevitable ageing of the satellites batteries is the main driver for further studies on the battery performance which leads to continuous updates on the Mission Planning System. A model that can accurately represent the battery performance, which will increase the efficiency of the battery utilization in the upcoming years for both TerraSAR-X and TanDEM-X satellites, is a great challenge for both the Power and Thermal team and the Mission Planning team. 


\section{Conclusion}

The TerraSAR-X satellite is running its $11^{\text {th }}$ year in orbit, followed by the TanDEM-X satellite in its $8^{\text {th }}$ year in orbit, both being in great condition and fully operational. The performance of the batteries of both satellites is as expected. The battery voltage minimum values are still far from the limits that would trigger an onboard event. Through continuous monitoring and performing various analyses we try to optimize the battery utilization, respecting all the constraints and requests of both missions on both satellites, for a safer and longer battery life. Furthermore, the information and experience gathered, as well as the studies and the models implemented, create the basis for a significant knowledge-input for current or upcoming missions that will deal with similar challenges on the satellite batteries in the future.

\section{Acknowledgments}

The Authors thank their colleagues from the German Space Operations Center for their engaged work in the TerraSAR-X/TanDEM-X daily operations, especially the Mission Planning team: Guillaume Guillermin, Ibrahim Koek, Nadine Hermannsdoerfer and Daniel Grinham, as well as the Power and Thermal team: Kay Mueller, Arvind Balan and Alice Bonfanti.

\section{References}

[1] “Dual Satellite Operations in Close Formation Flight”, E.Maurer, S.Zimmermann, F.Mrowka, H.Hofmann, Proceedings of the 12th International Conference on Space Operations (SpaceOps 2012), 11-15 June 2012, Stockholm, Sweden.

[2] “The Joint TerraSAR-X/TanDEM-X Mission Planning System”, F.Mrowka, M.P.Geyer, C.Lenzen, A.Spörl, T.Göttfert, E.Maurer, M.Wickler, B.Schättler, Proceedings of the International Geoscience and Remote Sensing Symposium (IGARSS 2011), 24-29 July 2011, pp. 3971-3974, Vancouver, Canada.

[3] C. Lenzen, Implementation of Power and Thermal Constraints in Mission Planning, TD-MOS-TN-3043, GSOC, DLR, Project internal document, 2010

[4] "Evolving the Operations of the TerraSAR-X/TanDEM-X Mission Planning System during the TanDEM-X Science Phase", F.Stathopoulos, G.Guillermin, C.G.Acero, K.Reich, F.Mrowka, Proceedings of the 14th International Conference on Space Operations (SpaceOps 2016), 16-20 May 2016, Daejeon, Korea.

[5] "Li-Ion Battery Operations and Life Optimization”, A.K.Balan, C.Lenzen, Proceedings of the 68th International Astronautical Congress (IAC), 26-30 September 2017, Adelaide, Australia.

[6] F. Schiemenz, TerraSAR/TanDEM-X Technical Note on Analysis of Battery Low Voltage Events, TSX-AED-TN-122, Project internal document, 2015

[7] K. Mueller, Technical Note on Assessment of Battery Degradation Effects and their Operational Handling, TX-GS-TN2016, Project internal document 